\section{Mesenchymal stromal cells and the acute respiratory distress syndrome (ARDS): challenges for clinical application}

\author{
J E Millar, ${ }^{1}$ J F Fraser, ${ }^{2}$ D F McAuley ${ }^{3}$
}

In contemporary reports, approximately $30 \%$ of patients with acute respiratory distress syndrome (ARDS) will die $^{1}$ and up to $70 \%$ of survivors have persistent significant disability. ${ }^{2}$ Several interventions which limit injurious ventilation have been shown to reduce mortality, primarily: low-tidal volume ventilation, ${ }^{3}$ prone positioning, ${ }^{4}$ early neuromuscular block$\operatorname{ade}^{5}$ and possibly extracorporeal membrane oxygenation (ECMO). ${ }^{6}$ To date, no intervention targeted at the underlying pathophysiological process has been shown to be beneficial, including recent studies of $\beta$-agonists and statins. ${ }^{78}$

Given the failure of pharmacological interventions in ARDS, increasing interest has been shown in the potential of mesenchymal stromal cells (MSCs). While the complex actions of MSCs are not yet fully understood, they appear to attenuate lung injury via three broad mechanisms. First, they have an immunomodulatory ability, influencing both innate and adaptive immunity. This is achieved by the secretion of anti-inflammatory soluble factors, including interleukin 10 (IL-10), IL-1 receptor antagonist and prostaglandin E2. ${ }^{9}$ Second, MSCs directly augment the host response to sepsis. LL-37 is a peptide secreted by MSCs which has direct antimicrobial properties and has previously been shown to increase bacterial clearance in an Escherichia coli model of lung injury after the intratracheal administration of MSCs. ${ }^{10}$ Third, MSCs play an important role in the repair and regeneration of lung tissue following injury. This ability appears to be mediated by the secretion of several growth factors, including vascular endothelial growth factor and keratinocyte growth factor. ${ }^{9}$

\footnotetext{
'Department of Anaesthesia, Critical Care \& Pain, University of Glasgow, Glasgow, UK; ${ }^{2}$ Critical Care Research Group, The Prince Charles Hospital and The University of Queensland, Brisbane, Queensland, Australia; ${ }^{3}$ Centre for Infection and Immunity, Queen's University Belfast, Belfast, UK

Correspondence to Dr D F McAuley, Centre for Infection and Immunity, Queen's University Belfast, Health Sciences Building, 97 Lisburn Road, Belfast BT9 7BL, UK; d.f.mcauley@qub.ac.uk
}

In this edition of Thorax, Devaney et $a l,{ }^{11}$ in an elegant series of experiments have contributed important new evidence supporting a potential role for MSCs in ARDS. The administration of human bone marrow-derived MSCs (hMSCs) resulted in improved lung injury in animals treated with MSC as compared with those treated with saline or fibroblasts. These treated animals exhibited lower rates of alveolar neutrophil infiltration and higher levels of IL-10, demonstrating the immunomodulatory properties of hMSCs. Crucially, this study also found that hMSCs act to reduce the bacterial burden and enhance the function of macrophages. These data support results obtained from previous work in both ex vivo and animal models of ARDS. ${ }^{12} 13$ Importantly, the authors also present results, which have direct relevance to our future ability to translate MSC therapy to patients. Their study compared the use of cryopreserved hMSCs against fresh cells and showed no difference in efficacy. The ability to preserve cells for storage or transport would increase the feasibility of MSCs as a therapeutic option. Furthermore, the study showed no advantage to intratracheal instillation when compared with intravenous administration. In the setting of significant hypoxia associated with ARDS, intravenous administration offers a safer and more convenient method of administration.

One factor to consider in the translation of these data relates to the relevance of data from small animal models to patients with ARDS. It is recognised that ventilation in rodents differs from humans ${ }^{14} 15$ and moreover genomic responses in mouse models correlate poorly with human inflammatory diseases. ${ }^{16}$ One approach to compliment and advance the data from small animal models is to use a large animal ovine model of ARDS, which most closely represents human pulmonary physiology. ${ }^{17}$

In a previous editorial, ${ }^{18}$ reviewing preclinical studies on MSCs in ARDS, ${ }^{13}{ }^{19}$ the argument was made that the time was appropriate for clinical trials of MSCs in patients with ARDS. Two phase I trials have been completed demonstrating safety in the acute setting, ${ }^{20} 21$ and two phase IIa studies are in progress: (1) Matthay et $a l^{22}$ are undertaking a randomised, doubleblind, placebo-controlled, multicentre trial, investigating a single dose of allogeneic bone marrow-derived MSCs (10 M cells $/ \mathrm{kg}$ ) in patients with ARDS in the USA (NCT02097641) and (2) Le Blanc and Grinnemo $^{23}$ are undertaking a nonrandomised open-label, controlled multicentre trial, investigating a single dose of allogeneic bone marrow-derived MSCs (2 $\mathrm{M}$ cells $/ \mathrm{kg}$ ) in patients with viral-induced ARDS on ECMO (NCT02215811). There is also significant pharma interest in cell therapy in ARDS. However, a number of key questions remain unanswered.

First, while the current study investigators have used bone marrow-derived MSCs, other groups have reported MSCs derived from different tissue types. ${ }^{9}$ MSCs derived from bone marrow present logistical challenges as a source of cells for large-scale clinical applications. MSCs derived from other tissue types, such as umbilical cord, may be a better source of cells. In addition to being a relatively unlimited tissue source, umbilical cord-derived MSCs may possess additional attributes, including greater anti-inflammatory potential and better proliferation kinetics. $^{24}$ Further clinical studies are required to determine the optimal MSC source.

The ARDS population to be studied in clinical trials merits further consideration; to date, preclinical studies of MSCs have focused on moderately severe models of lung injury. Future studies should also concentrate on testing the potential of MSCs in more severe ARDS where mortality is highest such as those patients receiving ECMO. Given the rapid expansion in the use of extracorporeal techniques, the interaction between these two therapies should be tested in preclinical studies to help inform the appropriate design of future clinical trials of MSCs in patients receiving ECMO. Patients receiving ECMO also offer a unique opportunity to investigate the pulmonary mechanisms by which MSCs act, through the ready access to bronchoalveolar lavage regardless of the severity of hypoxia. There may also be the opportunity to deliver MSCs to the preprepared ECMO circuit rather than the lungs per se, as the extracorporeal circuit is a site of substantial inflammatory excess related to the hyperoxic environment which exists there. Organisations such as ECMONet will be invaluable in facilitating such clinical trials (http://www.internationalecmonetwork. org). 
Finally, the challenges posed by the potential heterogeneity of donated cells and our lack of a potency assay to define a consistent final cell product, in addition to the issues of scaling cell manufacturing and distribution to supply multiple clinical sites and potentially thousands of patients, need to be considered. Research should focus on improving the isolation and definition of MSCs to reduce heterogeneity, which will be required to fulfil expected future regulatory requirements for cell therapy. ${ }^{25}$ In the UK, a recent Department of Health position paper on Regenerative Medicine $^{26}$ highlighted that the NHS Blood and Transplant (NHSBT), with its expertise in supplying safe blood and tissue products to hospitals across the UK, would make it a natural partner in any national regenerative medicine strategy. If cell therapies are proven to be effective, the infrastructure that bodies such as the NHSBT have in place will need to be used to ensure that cell therapies can be delivered as a standard of care.

In conclusion, MSC therapy holds the promise of becoming a revolutionary treatment for ARDS. Ongoing preclinical work, such as the work by Devaney et al, is needed to inform future clinical trials. The next challenges in clinical translation will be to deliver a consistent cell product, in a scalable manner, to treat the significant number of patients with ARDS who might benefit from this therapy. Patients with the most severe ARDS requiring ECMO may represent a group in which this promising therapy can provide important benefits.

Correction notice This article has been corrected since it was published Online First. The provenance and peer review statement has been corrected.

Contributors JEM wrote the initial draft. JFF and DFM edited and finalised the final version.

Competing interests DFM currently has a funding application under consideration for a trial of MSCs in ARDS in conjunction with Orbsen Therapeutics.

Provenance and peer review Commissioned; internally peer reviewed.

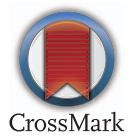

To cite Millar JE, Fraser JF, McAuley DF. Thorax 2015;70:611-612.

Published Online First 19 May 2015

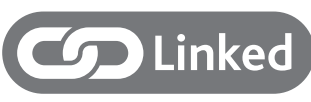

http://dx.doi.org/10.1136/thoraxjnl-2015-206813

Thorax 2015;70:611-612.

doi:10.1136/thoraxjnl-2015-207121

\section{REFERENCES}

1 Phua J, Badia JR, Adhikari NK, et al. Has mortality from acute respiratory distress syndrome decreased over time? a systematic review. Am I Respir Crit Care Med 2009;179:220-7.

2 Herridge MS, Cheung AM, Tansey CM, et al. Epidemiology and outcomes of acute lung injury. N Engl J Med 2003;348:683-93.

3 Serpa Neto A, Cardoso SO, Manetta JA, et al. Association between use of lung-protective ventilation with lower tidal volumes and clinical outcomes among patients without acute respiratory distress syndrome: a meta-analysis. JAMA 2012;308:1651-9.

4 Guerin C, Reignier J, Richard JC, et al. Prone positioning in severe acute respiratory distress syndrome. N Engl J Med 2013;368:2159-68.

5 Papazian L, Forel JM, Gacouin A, et al. Neuromuscular blockers in early acute respiratory distress syndrome. N Eng/ J Med 2010;363: 1107-16.

6 Peek GJ, Mugford M, Tiruvoipati R, et al. Efficacy and economic assessment of conventional ventilatory support versus extracorporeal membrane oxygenation for severe adult respiratory failure (CESAR): a multicentre randomised controlled trial. Lancet 2009;374:1351-63.

7 Gao Smith F, Perkins GD, Gates $S$, et al. Effect of intravenous $\beta$-2 agonist treatment on clinical outcomes in acute respiratory distress syndrome (BALTI-2): a multicentre, randomised controlled trial. Lancet 2012;379:229-35.

8 McAuley DF, Laffey JG, O'Kane CM, et al., HARP-2 Investigators; Irish Critical Care Trials Group. Simvastatin in the acute respiratory distress syndrome. N Engl J Med 2014;371:1695-703.

9 Curley GF, McAuley DF. Stem cells for respiratory failure. Curr Opin Crit Care 2015;21:42-9.

10 Krasnodembskaya A, Song $Y$, Fang $X$, et al. Antibacterial effect of human mesenchymal stem cells is mediated in part from secretion of the antimicrobial peptide LL-37. Stem Cells 2010;28:2229-38.

11 Devaney J, Horie S, Masterson C, et al. Human mesenchymal stromal cells decrease the severity of acute lung injury induced by $E$. coli in the rat. Thorax 2015;70:625-35

12 McAuley DF, Curley GF, Hamid UI, et al. Clinical grade allogeneic human mesenchymal stem cells restore alveolar fluid clearance in human lungs rejected for transplantation. Am J Respir Crit Care Med 2014;306:L809-15.

13 Curley GF, Hayes M, Ansari B, et al. Mesenchymal stem cells enhance recovery and repair following ventilator-induced lung injury in the rat. Thorax 2012;67:496-501.

14 Dunster KR, Friese M, Fraser JF, et al. Ventilation distribution in rats: Part I-The effect of gas composition as measured with electrical impedance tomography. Biomed Eng Online 2012;11:64.

15 Dunster $\mathrm{KR}$, Friese $\mathrm{ME}$, Fraser JF, et al. Ventilation distribution in rats: part 2-a comparison of electrical impedance tomography and hyperpolarised helium magnetic resonance imaging. Biomed Eng Online 2012;11:68.

16 Seok J, Warren HS, Cuenca AG, et al. Genomic responses in mouse models poorly mimic human inflammatory diseases. Proc Natl Acad Sci USA 2013;110:3507-12.

17 Asmussen S, Ito H, Traber DL, et al. Human mesenchymal stem cells reduce the severity of acute lung injury in a sheep model of bacterial pneumonia. Thorax 2014;69:819-25.

18 Mac Sweeney R, McAuley DF. Mesenchymal stem cell therapy in acute lung injury: is it time for a clinical trial? Thorax 2012;67:475-6.

19 Gupta N, Krasnodembskaya A, Kapetanaki M, et al. Mesenchymal stem cells enhance survival and bacterial clearance in murine Escherichia coli pneumonia. Thorax 2012;67:533-9.

20 Zheng G, Huang L, Tong H, et al. Treatment of acute respiratory distress syndrome with allogeneic adiposederived mesenchymal stem cells: a randomized, placebo-controlled pilot study. Respir Res 2014;15:39.

21 Wilson JG, Liu KD, Zhuo H, et al. Mesenchymal stem (stromal) cells for treatment of ARDS: a phase 1 clinical trial. Lancet Respir Med 2015;3:24-32.

22 Matthay MA. Human mesenchymal stem cells for acute respiratory distress syndrome (START). ClinicalTrials. gov, National Library of Medicine (US). https://clinicaltrials.gov/ct2/show/NCT02097641 (accessed 10 May 2015).

23 Grinnemo KH. Treatment of severe acute respiratory distress syndrome with allogenic bone marrowderived mesenchymal stromal cells. ClinicalTrials.gov, National Library of Medicine (US). https://clinicaltrials. gov/ct2/show/NCT02215811 (accessed 10 May 2015).

24 Jin HJ, Bae YK, Kim M, et al. Comparative analysis of human mesenchymal stem cells from bone marrow, adipose tissue, and umbilical cord blood as sources of cell therapy. Int I Mol Sci 2013;14:17986-8001.

25 British Standards Institute. PAS 93:2011 Characterization of human cells for clinical applications. http://shop.bsigroup.com/en/forms/PASs/ PAS-93/ (accessed 16 Apr 2015).

26 Department of Health. Taking Stock of Regenerative Medicine in the United Kingdom. https://www.gov. uk/government/uploads/system/uploads/attachment_ data/file/32459/11-1056-taking-stock-ofregenerative-medicine.pdf (accessed 16 Apr 2015). 